\title{
Fractured Identity: A Framework for Understanding Young Asian American Women's Self-harm and Suicidal Behaviors
}

\author{
Hyeouk Chris Hahm • Judith G. Gonyea • \\ Christine Chiao $\cdot$ Luca Anna Koritsanszky
}

Published online: 22 January 2014

(C) Springer Science+Business Media New York 2014

\begin{abstract}
Despite the high suicide rate among young Asian American women, the reasons for this phenomenon remain unclear. This qualitative study explored the family experiences of 16 young Asian American women who are children of immigrants and report a history of self-harm and/or suicidal behaviors. Our findings suggest that the participants experienced multiple types of "disempowering parenting styles" that are characterized as: abusive, burdening, culturally disjointed, disengaged, and gender-prescriptive parenting. Tied to these family dynamics is the double bind that participants suffer. Exposed to multiple types of negative parenting, the women felt paralyzed by opposing forces, caught between a deep desire to satisfy their parents' expectations as well as societal expectations and to simultaneously rebel against the image of "the perfect Asian woman." Torn by the double bind, these women developed a "fractured identity," which led to the use of "unsafe coping" strategies. Trapped in a "web of pain," the young women suffered alone and engaged in self-harm and suicidal behaviors.
\end{abstract}

This paper was presented at the 141st American Public Health Association Annual Meeting in Boston, MA, on November 4, 2013.

H. C. Hahm $(\bowtie)$ · J. G. Gonyea

Boston University School of Social Work, Boston, MA, USA

e-mail: hahm@bu.edu

J. G. Gonyea

e-mail: jgonyea@bu.edu

C. Chiao $\cdot$ L. A. Koritsanszky

Boston University School of Public Health, Boston, MA, USA

e-mail: cchiao@bu.edu

L. A. Koritsanszky

e-mail: 1koritsa@bu.edu
Keywords Asian American women - Suicide - Mental health $\cdot$ Self-harm $\cdot$ Parenting $\cdot$ Child abuse

\section{Introduction}

Suicide among Asian American women has emerged as a significant public health problem. In 2009, young Asian American women had the second highest rate of suicide among those aged 15-24 of all racial groups, after their Native American counterparts (National Center for Health Statistics 2012). This age group of Asian American women also showed rapid growth in incidence of suicide, with suicide mortality rate rising from 2.8 deaths per 100,000 in 2004 to 5.3 deaths per 100,000 in 2009 (National Center for Health Statistics 2012). This vulnerability extends across immigrant generations; U.S.-born Asian American women have been found to have higher rates of suicide ideation and suicide attempts compared to those who are foreign born (Duldulao et al. 2009).

Although the empirical literature exploring predictors of suicidality among Asian Americans is relatively sparse, emerging epidemiological literature suggests that poor family communication (Ying and Han 2007), the internalization of the model minority stereotype (Noh 2007), acculturative stress among family members (Duldulao et al. 2009), discrimination, poor family communication, and drug use (Hahm et al. 2013) are associated with suicidal ideation or suicide attempts. In particular, the poor family dynamic factor (e.g., poor family communication, negative parentchild interactions) was one of the most powerful and consistent correlates of suicide among Asian Americans in general (Cheng et al. 2010; Hahm et al. 2013; Wonget al. 2012). Given the strong emphasis that the Asian culture places on family unity and harmony (Tummala-Narra and Gaw 2000), it is intuitive that family dynamics is a critical 
factor for influencing health risk behaviors among young Asian American women.

Family dynamics both influence and are shaped by the nature of the "parenting style" and the quality of interactions between family members. Studies show that certain types of parenting styles have an association with suicide behaviors. Martin and Waite (1994) found that an "affectionless control" parenting style was correlated with an increased risk for suicidal thoughts among a predominately white Australian high school student sample. Among Asian populations, two studies of Chinese high school and college students found "authoritarian," "over-protective" or "over-control," and "low-warmth" or "low-affection" parenting to be significantly associated with greater likelihoods of suicide-related behaviors (Lai and McBride-Chang 2001; Gau et al. 2008). These findings suggest that understanding individuals in the context of their families, specifically the parenting styles that Asian American women experience, may be a critical component to reducing the rate of suicidal behaviors.

There are three methodological limitations in the current empirical literature that warrant further investigation on the role of parenting style. First, the majority culture's definitions of parenting styles, such as "authoritarian" or "authoritative" parenting, concepts based on North American developmental psychology (Baumrind 1971) may not be culturally relevant or appropriate to describe practices in Asian American families (Choi et al. 2013; Kim et al. 2013). Reframing these parenting styles in a culturally specific context is necessary to extend the implications within the unique dynamics of the Asian American family.

Second, there has been relatively little investigation of the specific mechanisms by which parenting styles are linked, either directly or indirectly, to self-harm and suicidal behaviors. Exploration of detailed stories or specific examples of how these parenting styles are perceived by Asian American women who were involved in self-harm and suicidal behaviors may deepen understanding of the short- and longer-term effects on mental health functioning. Third, although the process of identity formation has been linked to mental health outcomes (Meeus et al. 1999), how parenting style interplays with identity formation to affect mental health and suicidality among Asian American women has not been elucidated. To address these limitations, we used qualitative data from in-depth interviews with Asian American women who have reported self-harm or suicidal behaviors.

\section{Perceived Parenting, Identity Formation, and Suicidality Among Asian American Women}

Asian Americans are the fastest growing population in the U.S., and approximately $64 \%$ of Asians in the U.S. are foreign born (Gryn and Gambino 2012). Similar to other immigrant families in the U.S., immigrant Asian families face a multitude of challenges and often experience acculturative stress due to differences in language, culture, and values, as well as factors such as race/ethnic discrimination, job insecurity, and trauma histories (Sodowsky and Wai Ming Lai 1997). Among these challenges is a difference in expectations of parenting styles between parents, who retain more traditional beliefs of their homeland culture and their more acculturated children who are raised in America.

Chao (1994) argues that in Asian culture, the dominant parenting style is best characterized by a "training" ideology, which incorporates aspects of parental control, a high level of parental involvement in children's education, prioritizing obedience, continuous monitoring, and strict guidance of children's behaviors and conduct. Training ideology is the combination of deep care and involvement in the child's development, which fosters parents' positive relationship with the child. Choi et al. (2013) conceptualize Korean parenting as "ga-jung-kyo-yuk," in which core values are composed of the centrality of family, family hierarchy, respect, using appropriate etiquette with parents and others, and family obligations and ties.

Amy Chua's (2011) memoir, "The Battle Hymn of the Tiger Mother," is an extreme implementation of aspects of training ideology or "ga-jung-kyo-yuk," which accentuates harshness, strictness, and lack of family democracy; this negativity deviates from core aspects of training ideology and "ga-jung-kyo-yuk." Her memoir captures the use of "tiger mom parenting," which is based on the aforementioned "training" parenting ideology and utilizes intense parental monitoring, setting high expectations, and harshness in order to achieve self-discipline, self-reliance, and success in children's lives (Chua 2011). As an Asian parent herself, Chua comes to discover that utilization of the "training" parenting style can trigger much conflict in the family. In fact, the "tiger parenting" profile was associated with lower grade point average (GPA), lower sense of family obligation, higher level of academic pressure, higher level of depressive symptoms, and higher level of alienation among a sample of 444 Chinese American families (Kim et al. 2013).

Immersed daily in American culture at school and among their peers, Asian American children are exposed to two very different worldviews of "appropriate" parenting styles. "Training ideology" and "ga-jung-kyo-yuk" also manifest differentially in young males and females (Chao 1994; Choi et al. 2013). Tasked with the process of having to adjust to the normative gender roles of two very different cultures, Asian American women who were raised in "traditional" cultures that are more heavily gender differentiated than American culture often face further fracturing 
of the identity formation process (Goodenow and Espin 1993). Women's ability to reconcile these discrepancies may be supported or hindered by the nature or quality of their family dynamics. Thus, significant questions exist about the impacts of training-oriented Asian parenting style not only on children's emotional well-being, but also how such parenting affects women differentially, particularly within immigrant families experiencing high levels of acculturative stress. Immigrant parents struggle with a sense of loss related to economic hardship or lower socioeconomic status (Ying 1988), language difficulties, demotion of their status in the U.S. work force, divorce, and the traumatic histories in their home countries, or personal issues with mental illness or substance abuse (Zhang and Norvilitis 2002).

Therefore, children whose parents suffer from the aforementioned issues may be more vulnerable to developing adverse mental health consequences and may face challenges with healthy identity formation. Developing a healthy identity is critical for these individuals, because it can be used as a positive strategy to better cope with not only acculturative stress but also other life stresses (Sodowsky et al. 1995). However, developing a healthy identity requires supportive parenting (Beyers and Goossens 2008) as well as generational consonance that includes the sharing of common values, expectations for the future, and similar world views (Portes and Rumbaut 2001).

Studies indicate a link between poor identity formation and an array of mental health problems, including suicidal behaviors among youth. Erikson's theory on psychosocial development holds that personal identity formation is a key component of development occurring during adolescence, and suicidal behavior, in part, reflects disturbances in the identity formation process and failure to obtain a sense of self (Erikson 1968). Marcia (1989) further supports identity formation as an indicator of psychological well-being, as it is indicative of a person's ability to adapt to their environment. Similarly, the stage of the identity crisis during adolescence was found to be significantly associated with a lower level of psychosocial well-being, including depression and anxiety (Crocetti et al. 2008; Meeus et al. 1999). Schwartz et al. argue that what these theories have in common and simultaneously share with Asian American culture is the importance of values: the cultural values adolescents internalize from belonging to a group, as well as the personal values that guide personal identity formation (Schwartz et al. 2008). They have proposed that cultural identity is an essential component to the construct of personal identity. For Asian American women, cultural identity formation is a crucial process to the larger process of personal identity formation.

Through the voices of Asian American women who reported self-harm and suicidal behaviors and faced the complex issues associated with being children of immigrants, this current study sought to gain greater insight into the connection between family context, identity formation, and self-harm and suicidal behaviors. While literature on suicide among Asian American women is limited, literature on self-harm is even more scarce; yet, self-harm behavior is linked to suicidal attempts (Harriss and Hawton 2005; Hawton et al. 2003). We define self-harm as deliberate selfinjury without the explicit intent to kill oneself. As will be described in this article, our analysis of family contexts led us to propose a "fractured identify model" as a framework for understanding Asian American women's engagement in self-harm and suicidal behaviors.

The specific objectives of this study were twofold: (1) to describe the specific parenting style perceived by Asian American women who were engaged in these risk behaviors; and (2) to explore the psychological processes of these women and the possible relationship between perceived parenting style, identity development, and engagement in self-harm and suicidal behaviors.

\section{Methods}

The qualitative interviews are part of a larger mixed method study, the Asian American Women's Sexual Health Initiative Project (AWSHIP; www.bu.edu/awship) designed to examine parenting styles and identity formation among Chinese, Korean, and Vietnamese women who are children of immigrants. We chose to focus on these populations because they represent three of the four most prevalent Asian ethnic groups in Massachusetts (MA). (Among Asian Americans in MA, $35.4 \%$ are Chinese, $22.1 \%$ are Asian Indian, $14.3 \%$ are Vietnamese, and $7.3 \%$ are Korean.) Eligible participants for AWSHIP were: (1) unmarried women; (2) aged 18-35; (3) Chinese, Korean, Vietnamese, or a mix of these ethnicities; (4) 1.5 or 2nd generation immigrants; and (5) greater Boston area residents. A total of 547 women were screened for eligibility; $3.3 \%$ were ineligible and $5.1 \%$ did not follow through with the interview appointment after initial contact, resulting in 501 participants who were invited to participate in a computer-assisted self-interview (CASI) survey between January 2010 and July 2010. As part of the CASI survey consent process, individuals were asked whether they were interested in participating in future indepth interviews; approximately 497 (99\%) of survey participants answered affirmatively. These women formed the pool of potential participants for the AWSHIP qualitative study component.

Given the high prevalence of risky health behaviors found in the AWSHIP sample, a qualitative interview was undertaken to explore in greater depth the role of 
Table 1 Sample characteristics $(n=38)$

\begin{tabular}{|c|c|c|}
\hline Sample Characteristics & $n$ & $\begin{array}{l}\text { Prevalence } \\
(\%)\end{array}$ \\
\hline Total & 16 & 100.0 \\
\hline \multicolumn{3}{|l|}{ Ethnicity } \\
\hline Chinese & 7 & 43.7 \\
\hline Korean & 4 & 25.0 \\
\hline Vietnamese & 2 & 12.5 \\
\hline Mixed ethnicities & 3 & 18.8 \\
\hline \multicolumn{3}{|l|}{ Generation } \\
\hline 1.5 Generation (foreign born) & 3 & 18.8 \\
\hline 2nd Generation (U.S. born) & 13 & 81.3 \\
\hline Average age & $\begin{array}{l}22 \text { (Range: } \\
18-30)\end{array}$ & \\
\hline \multicolumn{3}{|l|}{ Education } \\
\hline High school diploma or less & 1 & 6.25 \\
\hline Some college or college degree & 12 & 75.0 \\
\hline $\begin{array}{l}\text { Graduate/professional school or } \\
\text { graduate degree }\end{array}$ & 3 & 18.8 \\
\hline \multicolumn{3}{|l|}{ Mother's education } \\
\hline High school diploma or less & 3 & 18.8 \\
\hline $\begin{array}{l}\text { College/professional school or } \\
\text { graduate degree }\end{array}$ & 12 & 75.0 \\
\hline Other & 1 & 6.25 \\
\hline \multicolumn{3}{|l|}{ Father's education } \\
\hline High school diploma or less & 2 & 12.5 \\
\hline $\begin{array}{l}\text { College/professional school or } \\
\text { graduate degree }\end{array}$ & 12 & 75.0 \\
\hline Other & 2 & 12.5 \\
\hline
\end{tabular}

acculturation on the occurrence of these behaviors, including self-harm and suicidality. Thus, we randomly selected a total of 23 women with varying levels of acculturation (as determined by their CASI survey acculturation scores) to be interviewed. During the initial interviews, we discovered that 8 out of 23 women $(35 \%)$ had been exposed to significant childhood or other life adversities. As a result, a decision was made to expand the sample size to also allow for analysis of these phenomena. Based on its use in prior research to understand health risks, we defined an "adversity history" as experiencing lifetime suicide attempts, self-harm, forced sex, sexual abuse, and illicit drug use (Biglan et al. 1990; Cheng et al. 2010; Hahm et al. 2011). Using these criteria, we select an additional eight women belonging to an adverse group and eight belonging to a non-adverse group to participate in qualitative interviews, effectively increasing the total qualitative study sample size to 38 women (Table 1).

This article is based on a subset of the 38 intervieweesspecifically, 16 women who self-identified as engaging in either self-harm and/or suicidal behaviors. As a result, lifetime suicide attempts and self-harm are the primary outcome for the preceding qualitative analysis. All of the qualitative interviews were conducted by three Asian American women with mental health expertise: the principal investigator (PI) who is a Korean American Ph.D. social work researcher and clinician, a Korean American anthropologist, and a Chinese American Masters of Social Work (MSW) intern. Using a semi-structured interview format, participants were asked about a variety of topics including their parents' immigration story, their own ethnic and racial identity, their family life growing up, their dating experiences, and sexual history. The interviews, which were conducted in English, lasted between 1 and $3 \mathrm{~h}$ (averaging $1.5 \mathrm{~h}$ ). Interviews took place either at the AWSHIP research center or at a location convenient for the participant, including other local colleges, public libraries, and their home. Informed consent was acquired, and interviews were audio recorded. The recordings were then transcribed verbatim. All digital recordings and transcripts were assigned a pseudonym to protect the participants' identities.

The PI and members of the research team listened to the digital recording and read the interview transcripts for the entire sample $(n=38)$. Since we were interested in analyzing only children of Asian immigrants, one participant was dropped because she was adopted by white parents.

We analyzed the interviews through an in-depth coding process based on content analysis. First, three research assistants were trained to code the entire 38 transcripts; these coders (Group 1) developed approximately 80 detailed coding categories and met with the first author to identify overlapping areas and develop a coding dictionary. The second coding group (Group 2) reviewed all cases $(n=38)$ to ensure consistency and accuracy of the coding dictionary developed by Group 1 and identified the final sample of 16 women who were involved in any self-harm and/or suicidal behaviors. Coding group 2 met together with the first author to address any new codes and category development. Next, the group moved to axial coding, in which they explored the relationship and connections between the generated thematic categories (Strauss and Corbin 1990). As part of this process of confirming the emerging patterns and themes, as well as identifying possible exceptions, the team continually compared and contrasted the 16 participants' narratives. Finally, a third group (Group 3) was trained in the coding system and independently reviewed both audio and transcripts to confirm validity of the thematic analysis. Group 3 focused on selective coding to determine the presence of a core of essential concepts contributing to the phenomena of selfharm and suicidal behavior. "Substance use," was defined as excessive drinking and substance use/abuse of hard drugs alone or in combination with soft drugs (Marijuana 


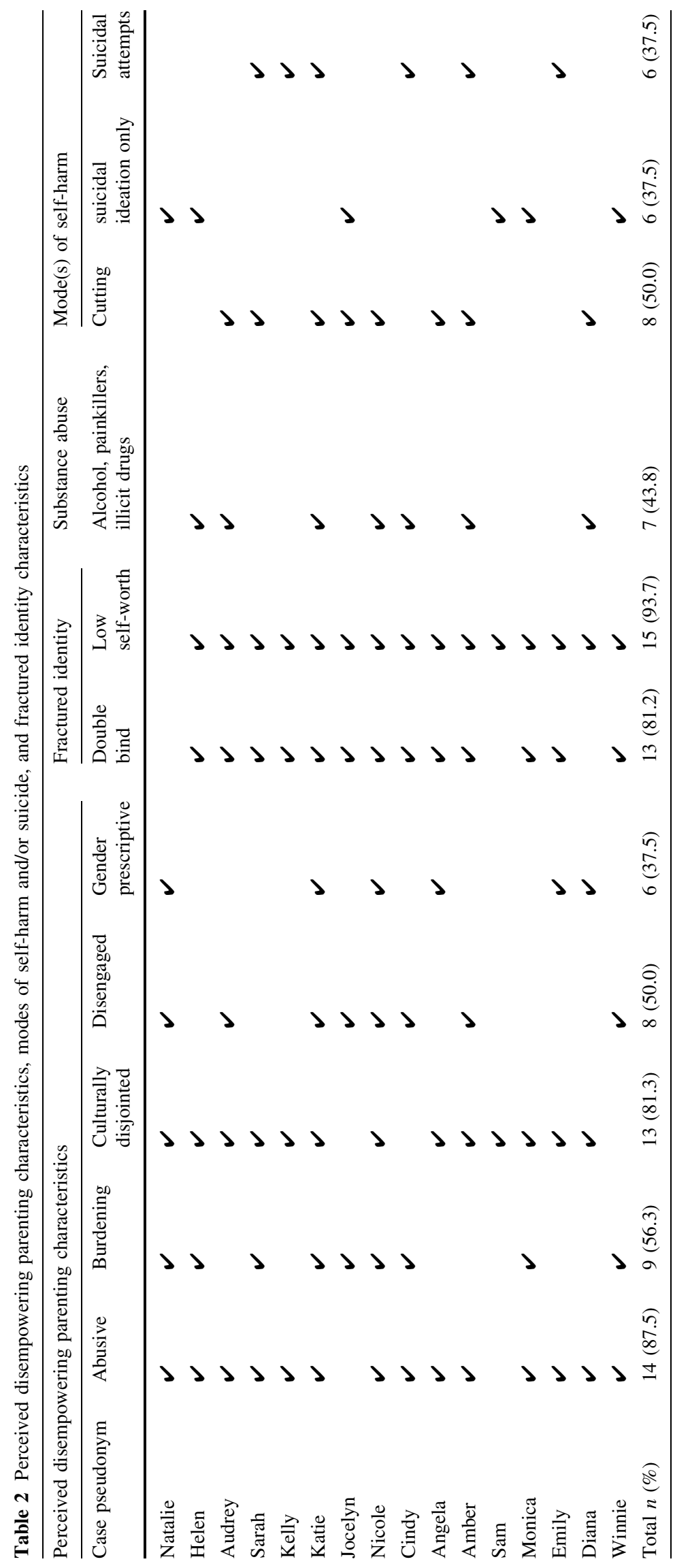


and smoking). All coders were blinded to participants' cutting and suicidal behaviors.

\section{Results}

The Process of Developing "Fractured Identity"

Analysis of categories led to the identification of "fractured identity" as the core concept, around which a narrative was built for understanding Asian American women's self-harm and suicidal behaviors. Our study suggests that young Asian American women born to immigrant parents practicing disempowering parenting styles are at greater risk of developing fractured identities. Our analysis of 16 participants reporting self-harm and suicidal behaviors resulted in the identification of five characteristics of "disempowering parenting styles" that are linked to the "lived" psychological experience of fractured identity and integrated in a phenomenon that we call the "web of pain." As shown in Table 2, all 16 women reported self-harm or suicidal behaviors; eight women who reported self-harm behaviors used "cutting" as the method for self-harm; six women reported suicidal ideation without any attempt; six women reported suicide attempt.

\section{Disempowering Parenting Characteristics}

We discovered five perceived parenting characteristics that represent a disempowering parenting style. These five characteristics include: abusive parenting, burdening parenting, culturally disjointed parenting, disengaged parenting, and gender-prescriptive parenting (thus termed "ABCDG parenting").

\section{(A) Abusive Parenting (Physical, Emotional, and Sexual Abuse)}

The majority of our participants reported that their parents used harsh discipline methods, and they experienced abuse: emotional (13 cases), physical ( 7 cases), sexual (3 cases), or a combination of at least two of these forms of abuse (7 cases). Emily (23, Chinese) describes an incident of sexual abuse from her father:

It was with my father... I guess it was like right after I had started like, developing breasts and, yeah. [Laughs]... this feels really weird to talk about. I mean, I guess like touching of like that area. I was awake.... I don't really remember, to be quite honest. I mean, I think it's one of those things I try to repress a little bit.

Angela (19, Vietnamese) described her father's prolonged emotional and physical abuse, which included beatings. Throughout her interview, Angela expressed a constant feeling of disempowerment and her only perceived way of regaining a sense of personal control was to self-harm:

My dad was definitely pretty physically abusive when I was growing up... I think that's where the cutting came from 'cause I was just angry that my dad was able to hurt me. So I kind of took it back in my own hands and would hurt myself on my own terms.

As commonly found in the abuse literature, all of the participants reporting physical abuse also identified experiencing emotional abuse. While physical abuse can leave visible marks or bruises, the effects of emotional abuse were just as damaging to these women, negatively impacting one's sense of self. For example, Cindy (28, Korean) felt hurt, humiliated, demoralized, and alienated by her mother's assumptions and comments about her promiscuity:

Like, even though I was a virgin, my mother was convinced that I was a slut. And she would constantly ask me these questions and be like, 'I know you're a whore.' I felt abandoned by my parents... I thought, 'Oh my God, if your own parents don't want you, what's wrong with you?'

\section{(B) Burdening Parents}

Nine out of the 16 participants in our study reported feeling tremendously burdened by their parents. This can be broken down into three distinct themes: (1) financial burden to repay parents for their investments in their daughter, (2) academic achievement, either by the daughters themselves or by proxy through an appropriate marriage to a husband who has attained high academic achievement, and (3) obligation to care for parents/elders.

For the daughters, these demands often felt one-sided in nature, serving parental needs. The societal expectation in Asian cultures describes an interdependent relationship, where parents sacrifice everything for their children (particularly for their education) under the expectation that this sacrifice will be returned when parents become old and dependent on their children. However, in these extreme applications of training ideology and Asian societal values, parents failed to evoke a sense of responsibility; instead, their claims for interdependence created an extreme burden, leaving daughters' sense of burden compounded with a sense of powerlessness.

One example of burdening parents is exemplified in the financial burden felt by Winnie (20, Chinese). She spoke of the constant pressure from her mother to pay back all the money that represented familial financial support: 
I owe her $\$ 150,000 \ldots$ it's no interest for the first three years and then after the third year if I haven't paid her back in full it's like $3 \%$ interest and then each year it increases by $1 \%$ and I pay to-and I have to pay back by-in 10 years.

Many of our participants reported suffering from such a sense of financial burden toward their parents and recognized that their American friends do not have the same "sense of debt."

Women interviewed also discussed the immense amount of pressure they felt from their parents to achieve "success," as described by the second theme of burdening parents. Whether that success was defined through academic or extracurricular achievements (particularly common in music endeavors), or through good marriages (financially successful man from a good family), participants felt that their parents' definitions were rigid and narrow. Specifically related to academic achievement, many participants felt a significant obligation to find success because their parents moved to the United States to give their children "better opportunities."

One such story comes from Audrey (19, Korean), who grew up with an emotionally and physically abusive father who had divorced three times since her mother passed away. Having to deal with this loss is compounded by her father's controlling and hypocritical attitudes. She says:

He had like my whole life planned, like what college I was going to go to, what I was going to do everything... the thing was because my dad's career isn't so stable, he expected me to just take care of all my school, you know, tuition, and everything... I am trying to live his dream and go to an expensive school, and pay for it myself.

She not only feels burdened by her father's imposition, she also feels bitter toward her father's hypocrisy and selfish attitudes; she perceives an imbalance between his expectations for her success and glory and his inability to support those ambitions, which are as much his dreams as they are hers.

Besides the pressure of repaying debt or being successful, many women felt a deep sense of responsibility to care for their parents, exemplifying the third theme of burdening parents. For example, Monica (21, Chinese) said that her father was "very Chinese" in his expectations of her when he was hospitalized with a terminal illness. Monica felt his expectations were unrealistic, yet felt guilty when she resisted his demands:

My dad was in the hospital for like two or three years...Every time he went into the hospital, he expected me to stay there the entire day even when I wasn't needed.... If I didn't stay there the whole time, he would be angry...I understand he was sick, but I couldn't stay $24 / 7$... there were times when I slept over at the hospital for like weeks.

These multiple types of burden, and the pressure placed on our study participants by their parents, often made the women feel trapped and as though they had "too many roles to fulfill."

\section{(C) Culturally Disjointed Parents}

Our participants frequently cited the acculturation gap between parents and children as an ongoing frustration and integral component of parent/child emotional disconnect. In addition to differing cultural expectations of the externalization of feelings and emotions, the language barrier can also play a major role in disjoint, as experienced by Monica: "My parents don't speak English or it's very limited. Even though I do speak Chinese, there are always like subtleties like emotional words that, you know, you may not be able to express in the language." The language barrier between parents and daughters was significant, but is not the only factor that creates cultural disjoint. Monica highlights her mother's "traditional mindset" as another contributing factor to the existing cultural disjoint between herself and her mother:

The reason why I'm studying Chinese is because I want to be able to communicate [with my parents], more effectively. But just the mindset, especially my mom, the mindset she has is very traditional and very close-minded and hard to just explain things to in general.

\section{(D) Disengaged Parents}

Another common theme among the participants was the lack of parental validation of their own emotions and feelings; this style of disengaged parenting differentiates from emotional maltreatment, in which children are subjected to extreme blaming, direct terrorizing and hostility, or exploitation (Trickett et al. 2011). Rather, their feelings were repeatedly dismissed, belittled, or ignored by the disengaged parents.

Several participants discussed parents who "seemed not to care" or who appeared to be totally emotionally distanced from their daughters: their parents were physically present, but psychological absent from their daughters' lives. This profound sense of alienation felt by the daughters was extremely damaging and hurtful to them as children, especially in comparison with other American families of their peers. Audrey felt that her father was particularly disengaged: 
I want to have like a closer relationship with my father...I want to, grow that bond, you know, but then I think of how he doesn't understand me as much and how, when I was in high school, he wasn't really there for me to really accept... that I wanted to go my own path. So when I think about that, I get very angry and want to stay away from him.

Similarly, Monica recalled, "I never felt like they really praised me or that they were proud of me. That definitely affected my sense of worth." Feelings of inadequacy are deeply engrained in our participants whose parents seemingly distanced themselves from their daughters; whether these sentiments would have been less extreme if these women's parents were raising boys instead of girls brings us to the next perceived parenting characteristic.

\section{(G) Gender-Prescribed Parenting}

Five of our study participants perceived gender-prescribed parenting while growing up. Originating from the cultural prescriptive role of gender, these parents hold very rigid and strict views on how the boys and girls should behave. Feeling "less valued than their brother," participants perceived differential treatment from their parents, citing "unfair treatment" that made them feel "powerless." The most common example expressed by our participants was that of rigid parenting.

One participant, Diana (25, Chinese), said, "My parents are a lot stricter with me than my older brother. And I always thought that was like, such utter crap...my parents were a lot more protective of me than of my brother.... I was definitely treated differently." Although this style of parenting may have been well intentioned, our participants perceived it as creating a familial gap that they were never able to overcome. Similarly rooted in her parents' biased gender prescription, Kelly (22, Vietnamese), experienced rigid parenting: "There was all sorts of limits of what I could and couldn't do, who I should socialize with... the time that I have that's actually free where I' $m$ not studying or doing activities should be my own-and who I want to talk with."

\section{The Double Bind}

As shown in Table 2, 13 of the 16 high-risk participants displayed the recurring theme of a "double bind," or the psychological confrontation of two opposing forces, where individuals are subject to two contradictory rules or standards, neither of which the individual can exhibit any control over. Our participants suffer from culturally defined opposing phenomena: the desire for approval and affirmation from parents and the desire to pull away, reject, or rebel from those same pressures. Pushing against either force in an attempt to practice self-autonomy results in a heavy psychological toll, including a deep sense of emotional discomfort and guilt for violating familial or social norms. When faced with such internal adversity of two non-separable forces that intersect and interplay, the human tendency is to dichotomize in an attempt to escape the resulting double bind. As a result, participants experience a fracturing of the self and are unable to incorporate either aspect of the double bind wholly into their personal identity during the adolescent identity formation process (Jamieson 1995).

Suffering from a sort of "perfect Asian woman syndrome," our participants desperately strive to be the ideal daughter and wife, and practice Asian cultural values. The culture-bound symptoms of this syndrome often included attending an Ivy League school, becoming or marrying a doctor and being a "dutiful daughter" (i.e., abstains from premarital sex, does not use drugs, and honors her debt to her parents). Daughters strive desperately to fulfill the role of "a perfect Asian woman" despite deep anger and resentment toward their parents, as well as feeling intensely invalidated by them.

Despite this, they dissolutely want to reject and condemn the image of the "perfect Asian woman" and become resentful of the whole notion of Asian culture. They feel suffocated by the idealized Asian woman image and wished to escape these norms and instead follow their own desires, a path that often leads to involvement in risky health behaviors, including unsafe sexual behaviors and/or drug use.

This internal conflict, or "double bind," was articulated by Audrey. She discusses how this inner conflict was externalized in her life by noting that, "You know, we've all had sex but we don't talk about it because they want to be viewed as that like perfect Korean child... would not have sex, would wait till marriage, umm, will not do drugs, will just be an angel." Audrey also talked about how much pressure she received from her father to succeed in school. Though initially a hard worker, she "rebelled": she had 55 absences from school, started using ecstasy fairly regularly, and she fell in love with a guy who is "everything opposite of what a Korean son should be." Like other participants, she strongly resents the Korean culture, one in which "women are in a lower class than men, and women always gossip." Yet, at the same time, she discusses becoming a mother who exemplifies "a perfect image of a woman," with a husband who is also from Korean culture.

\section{Low Self-Worth}

Table 2 exhibits that a struggle with low self-worth was evident in most of our participant's stories; 15 of the 16 high-risk participants reported low self-worth. Sarah (20, 
Chinese) focused heavily on the pieces of her life she was not satisfied with:

In eighth grade, I focused on my appearance, what I didn't like about myself, and as I got older, it's been more like I can never do enough or be good enough...I think overall, and for my parents especially, I probably set impossibly high standards for myself. Senior year, when I didn't get into my top colleges, it was hard for me to take-my selfworth and identity as being academically strong faltered.

Similarly, Kelly described rebelling and talking back to her parents to gain more freedom in high school; yet, she still secretly grappled with low self-esteem and a lack of assertiveness:

I felt like I was never good enough. Like I was a perfect student - but you know, you want me to do you want me to be a nun on top of it, you want me to go to church at six o'clock every day, you want me to do this, you want me to do this - the list was endless so I felt very insufficient.

Bound yet torn by two opposing forces, these women are unable to successfully integrate the facets of their identities as "good enough daughter," "good enough woman," "good enough Asian" or "good enough American." Instead, within this struggle, each woman appeared to have cultivated a poor self-image and low self-worth; they were filled with a sense of "never being good enough," resulting from an inability to assert their own sense of autonomy.

\section{Fractured Identity}

As they grow up, Asian American females often find themselves navigating between two distinct, very different, and often opposing cultural worlds, particularly in terms of gender role expectations and familial norms and obligations. Most find synergy or a way to integrate these two cultural forces; some women, however, remain unsettled or unbalanced. This was particularly evident in participants who grew up with parents exhibiting disempowering parenting styles, where the differences between the desire for parental approval and desire to pull away and rebel from those pressures were more pronounced. These women formed, at best, a "fractured identity" which ultimately led to a sense of low self-worth or the feeling that "I am never good enough" (Fig. 1).

\section{Substance Use}

Seven of our 16 participants reported substance abuse ranging from excessive alcohol consumption to illicit drug

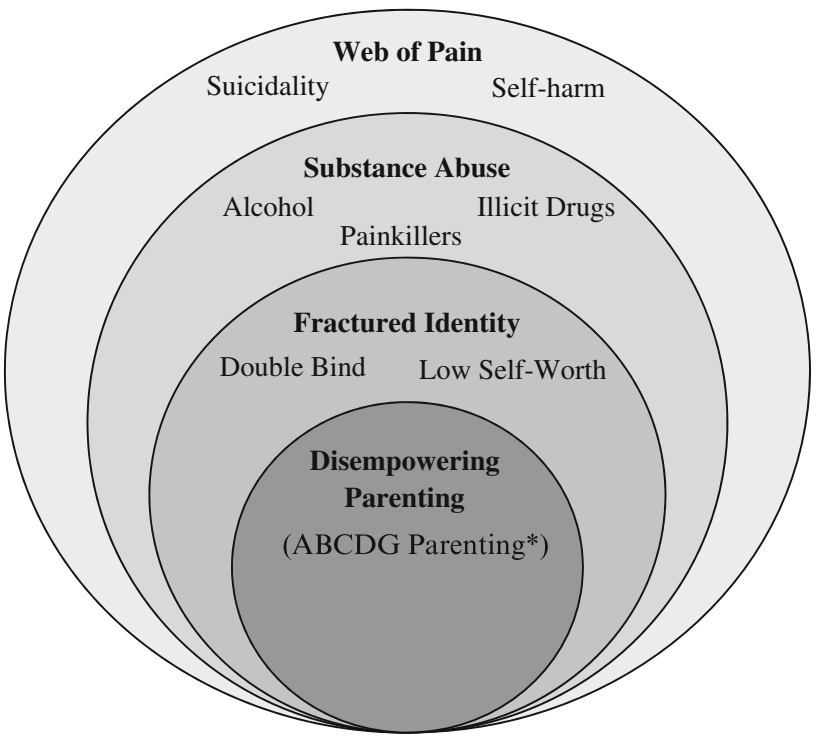

Fig. 1 Fractured identity model $*$ ABCDG parenting: $A$ abusive parenting, $B$ burdening parenting, $C$ culturally disjointed parenting, $D$ disengaging parenting, $G$ gender-prescribed parenting

use, including cocaine, mushrooms, painkillers, or ecstasy. Exemplifying this common thread throughout the subset of substance users, Diana (25, Chinese) states, "It [ecstasy] was fun because, like when you're on it you're just happy. So no matter what happens at like at home, or whatever happened at school, you're just happy."

As a common unsafe coping mechanism for participants exhibiting parasuicidality, participants reported using alcohol to "boost their self-esteem," or because "right now it's kind of like a solution" in addition to just evoking careless feelings of happiness. Here, the focus was on substance use as solving a "problem," as Audrey (19, Korean) states:

Umm, Ecstasy was a drug I did quite often... when I started doing drugs, that's when it was like the worst time of my life, when I like rebelled from my family and didn't want to go to school, so turning to drugs made me really happy. You know, I had no problems. I didn't think of any problems while on drugs. Umm, it seemed like all my problems were solved.

Our participants' relationship with substance use suggests that this phenomenon plays a significant role in the proposed causal pathway from disempowering parenting to suicidality.

\section{Web of Pain}

The consequences of fractured identity are expressed as psychological pain, despair, and emotional paralysis and numbness. Among our participants, this pain was expressed through self-harm behaviors, suicidal ideation, or suicide attempts. We call this phenomenon "web of pain" because these women often suffer alone and feel immobilized, stuck 
in the lives they are leading. At this point, it is necessary for them to seek professional help; however, they are reluctant to seek treatment or accept help from friends, family, or health professionals.

\section{Cutting to Achieve Self-control}

Among our participants, cutting was the most frequent method used to inflict self-harm, an act that was often viewed by participants as a way to regain or assert control. Both Ingrid and Naomi expressed this view of cutting as taking control, whether as an act of rebellion or self-punishment. Angela states,

Cutting was kind of like a release of tension and it gave me control again... I was just like angry at my parents, so it was a way of almost like getting back at them. It was something they didn't know about-it was like my own way of rebelling against them.

Naomi also expressed that her cutting was associated with her mother's abusive behaviors. "There's a Chinese saying for 'hit your own mouth'-my mom would make me punish myself; or else, she'd threatened to hit me herself.... When I got older, I turned to cutting as selfinflicted punishment. I thought I wasn't good enough."

\section{Suicidal Attempts and Invalidation from Parents}

Out of anger, desperation, and sense of helplessness, women may finally take action to end their lives; however, their attempts fail to offer emotional relief, often exacerbating a sense of invalidation from their own parents. Six women reported attempting suicide. One participant, Amber (27, Chinese), struggled greatly with suicide attempts and the connection between her family dysfunction:

My mom thought [my suicide attempt] was a 'silly' thing to do. At first [after my suicide attempt], I had this hope because my mom agreed that she'd never be mean to me anymore, that we'd talk about things and she would be my friend, but it just didn't work...she went back to her old ways. I could tell she didn't understand where I was coming from and so I just couldn't talk to her about it at all. And every time I try to tell her how unhappy I am, she just brushes it off like it's nothing, like it's not important...that I every negative thing I think is so trivial and so small...

Kelly, too, described how her father responded to her suicide attempt with anger and invalidation:

I tried to hang myself once after a big fight with my parents about the guy I was dating...but my father came home and found me tied to a rafter, already passed out...The next day I got the lecture about "you're going to hell."

\section{Suffering Alone}

The women who disclosed a history of self-harm described cutting as a way to objectify their pain while trying to keep it a secret. When Angela's school friends noticed her scars, confronted her and told her mother, she experienced mixed feelings about getting help:

It was kind of embarrassing 'cause it was like my thing and I didn't want people to know about it in the first place... and I didn't like being outed. But at the same time I guess I felt a little bit of relief knowing that someone else knew about this burden that I'd been carrying.

Monica also feels that there is a "stigma" against getting help. Similarly, Nicole (20, Chinese) was also trying to hide her cutting behaviors. She said, "My doctor kind of noticed that there were these scars on my arm, but I never told anyone except for like very close friends, just one or two of them." All of the participants generally hid their self-harm or suicidal behaviors from others or, if revealed to their parents, found little support. These women were isolated and alone in their webs of pain.

\section{Discussion}

Our fractured identity model describes the process by which Asian American women raised by "disempowering parents" are at risk of developing a "fractured identity," a phenomenon that may promote self-harm and suicidal behaviors. We specifically describe the five disempowering characteristics as "ABCDG parenting": abusive parenting, burdening parenting, culturally disjointed parenting, disengaged parenting, and gender-prescriptive parenting.

Objectively, the participants are accomplished young women, yet we found little evidence outlining a sense of positive self-worth or high self-esteem. Rather, the participants' voices largely reflect the constant struggles with the unresolved issues, including which culture they belong to, why they cannot have "normal" relationships with their parents, how to reduce their emotional pain and numbness, and how to simultaneously fit into both American and Asian cultural peer groups. Suffering from the "double bind," they are caught in an internal struggle between two opposing forces: the anger and antagonism toward their parents do not necessarily eliminate their sense of duty in fulfilling the expected role of being a "perfect daughter" 
or a "perfect woman." Therefore, when judged by Asian or American standards, participants continue to find themselves having "low self-worth"; lacking genuine emotional support and connection with their parents, participants never seem to hone in on an integrated sense of self.

Their resulting "fractured identity" traps these women in a web of pain, where they suffer alone, immobilized to get help. Because they feel invalidated by their own parents even after their suicide attempts, participants report powerlessness in their inability to justify their feelings and emotional pain. Among our sample, cutting was the most common self-harm behavior. This is true in the general U.S. population (Welch 2001) as well as in Asian countries (Shek and Yu 2012). Furthermore, health services research indicates English language use and health insurance as important enabling factors in seeking mental health care (Scheppers et al. 2006). Our participants were born in either the U.S. or immigrated to the U.S. when they were children; this means that English language proficiency is not an issue in seeking psychotherapy. Rather, feelings of helplessness resulting from disempowering parenting, combined with the shame and stigma associated with seeking professional help, drive them to suffer alone instead of seeking external outlets or professional help (Gilbert et al. 2007).

It is important to emphasize that immigrant parents often struggle intensely in their host social context. Many suffer through a sense of loss due to issues such as economic hardship, language difficulties, demotion of their status in the U.S. work force, divorce, traumatic histories in their home countries, or personal issues with mental illness or substance abuse. Considering the strong emphasis that Asian culture places on family unity and harmony- which can be unbalanced by the aforementioned hardships - it is possible that disempowering parenting styles may be a biproduct of Asian immigrant parents' overcompensation to restore balance in family dynamics in response to broader social and cultural challenges that Asian American immigrant parents often face.

A follow-up analysis was conducted to discern any similarities or differences between disempowering parenting components in our core sample of 16 participants who reported self-harm/suicidal behaviors and the group which did not report any of self-harm/suicidal behaviors. Compelling differences between the groups were evident in three parenting characteristics: abusive, burdening, and disengaged parenting. Abusive parenting was substantially higher among the self-harm/suicidal participants compared to their counterparts (87.5 vs. $19.0 \%$, respectively), followed by burdening (56.3 vs. $28.6 \%$ ) and disengaged (50.0 vs. $19.0 \%)$. Culturally disjointed and gender-prescriptive parenting, however, had similar patterns in the two groups. Despite these similarities, we continued to classify these two parenting styles as disempowering because they were perceived to be so among our self-harm/ suicidal participants. One possible explanation is that there may be an interactive effect between these culturally relevant parenting styles and the three other disempowering parenting styles (abusive, burdening, and disengaged) among the self-harm/suicidal participants. In other words, it is possible that the culturally disjointed and gender-prescriptive parenting may serve as a platform for or amplify the disempowering aspects of the other three negative parenting styles among women.

This study has some limitations related to the data. First, the sample size is small and limited to children of immigrants from three countries: China, Korea, Vietnam, and mix of the ethnic groups of these countries. Thus, this study cannot be generalized to all Asian American women, as there exists tremendous diversity among ethnic subgroups of Asian Americans in acculturation level, language, and socioeconomic status (Chen 2005; Zhang 2013). Second, our study is cross-sectional and retrospective in nature; therefore, we cannot predict whether low-risk women would later develop self-harm or suicidal behaviors. A longitudinal study is necessary to increase confidence in relationship between the disempowering parents, the development of fractured identity, and the involvement with self-harm and suicidal behaviors among this population. Third, readers should be cautious to not generalize our findings to all Asian American parenting, as our sample was confined women at high risk of mental health problems who have reported suicidal and self-harm behaviors. Similarly, this study is not to test whether the disempowering parenting styles are higher among Asian Americans compared to other ethnic groups. In fact, analysis by Kim et al. (2013) of 444 Chinese American families found that the "supportive parenting" profile was the most common, whereas the "harsh parenting" profile made up the smallest proportion in the sample.

Despite these limitations, our study provides important implications in research, practice, and policy. First, our study found that all our participants experienced multiple types of disempowering parents' characteristics, raising the question of whether the cumulative effects of disempowering parenting characteristics may be more harmful than women who experienced only one type of these characteristics. Our study results posit that disempowering parenting characteristics may serve as the mechanism toward the development of the fractured identity in women. This calls for the development of measures of disempowering parenting characteristics and components of the fractured identity phenomenon in future studies.

Second, our study suggests that helping women to develop a sense of empowerment within the family may be the key element for prevention of suicidal or self-harm 
behaviors among young Asian American women. This requires policy makers to pay more attention to creating resources for Asian American families to better deal with acculturative stress, language difficulties, and other unique needs as immigrants. Mental health services targeting Asian American families should take a more active role in reaching out to the children of immigrants through providing depression screening and access to mental health services that are culturally sensitive and specific to Asian American families.

Third, the study suggests the need for mental health professionals, educators, church, and other leaders in the Asian American community to promote a broadening of immigrant parents' definitions of "success." Through their words, our study participants revealed their constant struggle to be "the perfect daughter" or the "perfect woman." It becomes evident that definitions of "success" across families of immigrant parents and Asian American children are inextricably tied to academic or professional achievement, neglecting other aspects of emotional development. A focus on cultivating not only "academic intelligence," but also "emotional intelligence" is required. Emotional intelligence emphasizes social emotional learning as well as regulating emotions that promote resilience against unsafe coping, such as the high prevalence of substance abuse found in our final 16 sample of women exhibiting suicidal and self-harm behaviors (Coleman 2000).

Our study suggests that suicidal prevention/intervention for Asian American women should also focus on increasing family connection and bonding, parental validation of children's emotions, and cross-generational communication and understanding. Also key is addressing the stigma and shame associated with depression and suicide and breaking the silence of suffering alone. Targeting these components in prevention and intervention may lead Asian American women to help formulate a more integrated and solidified identity, ultimately breaking the web of pain and preventing self-harm and suicidal behaviors.

Acknowledgments This study was supported by grants $1 \mathrm{~K} 01 \mathrm{MH}$ 086366-01A1, a Mentored Research Scientist Development Award, and 1R34MH099943-01A1, a Clinical Trial Planning Grant, both from the National Institute of Mental Health

\section{References}

Baumrind, D. (1971). Current patterns of parental authority. Developmental Psychology, 4(1p2), 1. doi:10.1037/h0030372.

Beyers, W., \& Goossens, L. (2008). Dynamics of perceived parenting and identity formation in late adolescence. Journal of Adolescence, 31(2), 165-184. doi:10.1016/j.adolescence.2007.04.003.
Biglan, A., Metzler, C. W., Wirt, R., Ary, D., Noell, J., Ochs, L., et al. (1990). Social and behavioral factors associated with high-risk sexual behavior among adolescents. Journal of Behavioral Medicine, 13(3), 245-261. doi:10.1007/BF00846833.

Chao, R. K. (1994). Beyond parental control and authoritarian parenting style: Understanding Chinese parenting through the culturalnotion of training. Child Development, 65(4), 11111119. doi:10.1111/j.1467-8624.1994.tb00806.x.

Chen, M. S. (2005). Cancer health disparities among Asian Americans. Cancer, 104(S12), 2895-2902. doi:10.1002/cncr.21501.

Cheng, J. K. Y., Fancher, T. L., Ratanasen, M., Conner, K. R., Duberstein, P. R., Sue, S., et al. (2010). Lifetime suicidal ideation and suicide attempts in Asian Americans. Asian American Journal of Psychology, 1(1), 18-30. doi:10.1037/ a0018799.

Choi, Y., Kim, Y. S., Kim, S. Y., \& Park, I. J. K. (2013). Is Asian American parenting controlling and harsh? Empirical testing of relationships between Korean American and Western parenting measures. Asian American Journal of Psychology, 4(1), 19-29. doi: $10.1037 / \mathrm{a} 0031220$.

Chua, A. (2011). Battle hymn of the tiger mother. New York: Bloomsbury Publishing.

Coleman, D. (2000). Working with emotional intelligence. New York, NY: Bantam Books.

Crocetti, E., Rubini, M., Luyckx, K., \& Meeus, W. (2008). Identity formation in early and middle adolescents from various ethnic groups: From three dimensions to five statuses. Journal of Youth and Adolescence, 37(8), 983-996. doi:10.1007/s10964007-9222-2.

Duldulao, A. A., Takeuchi, D. T., \& Hong, S. (2009). Correlates of suicidal behaviors among Asian Americans. Archives of Suicide Research, 13(3), 277-290. doi:10.1080/13811110903044567.

Erikson, E. H. (1968). Identity: Youth and crisis. W. W: Norton \& Company.

Gau, S. S., Chen, Y. Y., Tsai, F. J., Lee, M. B., Chiu, Y. N., Soong, W. T., et al. (2008). Risk factors for suicide in Taiwanese college students. Journal of American College Health, 57(2), 135-142. doi:10.3200/JACH.57.2.135-142.

Gilbert, P., Bhundia, R., Mitra, R., McEwan, K., Irons, C., \& Sanghera, J. (2007). Cultural differences in shame-focused attitudes towards mental health problems in Asian and NonAsian student women. Mental Health, Religion \& Culture, 10(2). doi:10.1080/13694670500415124.

Goodenow, C., \& Espin, O. M. (1993). Identity choices in immigrant adolescent females. Adolescence, 28(109), 173-184.

Gryn, T., \& Gambino, C. (2012). The foreign born from Asia: 2011 (American Community Survey Briefs). Washington, DC: U.S. Department of Commerce, Economics and Statistics Administration.

Hahm, H. C., Jang, J., Vu, C., Alexander, L. M., Driscoll, K. E., \& Lundgren, L. (2013). Drug use and suicidality among Asian American women who are children of immigrants. Substance Use \& Misuse, 130712104413000. doi:10.3109/10826084.2013. 808219.

Hahm, H. C., Lee, C. H., Choe, J., Ward, A., \& Lundgren, L. (2011). Sexual attitudes, reasons for forgoing condom use, and the influence of gender power among Asian American women: A qualitative study. Journal of AIDS and clinical research, (S1). doi:10.4172/2155-6113.S1-004.

Harriss, L., \& Hawton, K. (2005). Suicidal intent in deliberate selfharm and the risk of suicide: The predictive power of the Suicide Intent Scale. Journal of Affective Disorders, 86(2-3), 225-233. doi:10.1016/j.jad.2005.02.009.

Hawton, K., Zahl, D., \& Weatherall, R. (2003). Suicide following deliberate self-harm: Long-term follow-up of patients who 
presented to a general hospital. The British Journal of Psychiatry, 182(6), 537-542. doi:10.1192/02-540.

Jamieson, K. H. (1995). Beyond the double bind. New York, NY: Oxford University Press.

Kim, S. Y., Wang, Y., Orozco-Lapray, D., Shen, Y., \& Murtuza, M. (2013). Does "tiger parenting" exist? Parenting profiles of Chinese Americans and adolescent developmental outcomes. Asian American Journal of Psychology, 4(1), 7-18. doi:10.1037/ a0030612.

Lai, K. W., \& McBride-Chang, C. (2001). Suicidal ideation, parenting style, and family climate among Hong King adolescents. International Journal of Psychology, 36(2), 81-87.

Marcia, J. E. (1989). Identity and intervention. Journal of Adolescence, 12(4), 401-410. doi:10.1016/0140-1971(89)90063-8.

Martin, G., \& Waite, S. (1994). Parental bonding and vulnerability to adolescent suicide. Acta Psychiatrica Scandinavica, 89(4), 246-254. doi:10.1111/j.1600-0447.1994.tb01509.x.

Meeus, W., Iedema, J., Helsen, M., \& Vollebergh, W. (1999). Patterns of adolescent identity development: Review of literature and longitudinal analysis. Developmental Review, 19(4), 419-461. doi:10.1006/drev.1999.0483.

National Center for Health Statistics (NCHS). (2012). Health, United States, 2011: With special feature on socioeconomic status and health. Hyattsville, MD. 2012. Retrieved from http://www.cdc. gov/nchs/data/hus/hus11.pdf.

Noh, E. (2007). Asian American women and suicide. Women and Therapy, 30(3-4), 87-107. doi:10.1300/J015v30n03_08.

Portes, A., \& Rumbaut, R. G. (2001). Legacies: The story of the immigrant second generation. California: University of California Press.

Scheppers, E., van Dongen, E., Dekker, J., Geertzen, J., \& Dekker, J. (2006). Potential barriers to the use of health services among ethnic minorities: A review. Family Practice, 23(3), 325-348. doi:10.1093/fampra/cmi113.

Schwartz, S., Zamboanga, B., \& Weisskirch, R. (2008). Broadening the Study of the Self: Integrating the study of personal identity and cultural identity. Social and Personality Psychology Compass 2/2 (pp. 635-651). doi:10.1111/j.1751-9004.2008.000777.

Shek, D., \& Yu, L. (2012). Self-harm and suicidal behaviors in Hong Kong adolescents: Prevalence and psychosocial correlates. The Scientific World Journal. doi: 10.1100/2012/932549.

Sodowsky, G. R., Kwan, K.-L. K., \& Pannu, R. (1995). Ethnic identity of Asians in the United States. In J. G. Ponterotto, J. M. Casas, L. A. Suzuki, \& C. M. Alexander (Eds.), Handbook of multicultural counseling (pp. 123-154). Thousand Oaks, CA, US: Sage Publications Inc.
Sodowsky, G. R., \& Wai Ming Lai, E. (1997). Asian immigrant variables and structural models of cross-cultural distress. In A. Booth, A. C. Crouter, \& N. S. Landale (Eds.), Immigration and the family: Research and policy on U.S. immigrants (pp. 211-234). Hillsdale, NJ, England: Lawrence Erlbaum Associates Inc.

Strauss, A., \& Corbin, J. (1990). Basics of qualitative research: Grounded theory procedures and techniques. Newbury Park, CA: Sage.

Trickett, P. K., Kim, K., \& Prindle, J. (2011). Variations in emotional abuse experiences among multiply maltreated young adolescents and relations with developmental outcomes. Child Abuse and Neglect, 35(10), 876-886. doi:10.1016/j.chiabu.2011.08.001.

Tummala-Narra, P., \& Gaw, A. P. (2000). Understanding family, community, immigration, and acculturation issues in psychodynamic psychotherapy with Asian Americans. Journal of psychiatric practice, 6(2), 69-76. doi:10.1097/00131746-20000300000001.

Welch, S. (2001). A review of the literature on the epidemiology of parasuicidality in the general population. Psychiatric Services, 52(3), 368-375.

Wong, Y. J., Uhm, S. Y., \& Li, P. (2012). Asian Americans family cohesion and suicide ideation: Moderating and mediating effects. American Journal of Orthopsychiatry, 82(3), 309-318. doi:10. 1111/j.1939-0025.2012.01170.x.

Ying, Y. W. (1988). Depressive symptomatology among ChineseAmericans as measured by the CES-D. Journal of Clinical Psychology, 44(5), 739-746. doi:10.1002/1097-4679(198809) 44:5<739:AID-JCLP2270440512>3.0.CO;2-0.

Ying, Y. W., \& Han, M. (2007). The longitudinal effect of intergenerational gap in acculturation on conflict and mental health in Southeast Asian American adolescents. American Journal of Orthopsychiatry, 77(1), 61-66. doi:10.1037/00029432.77.1.61.

Zhang, W. (2013). Asian-Americans, social-economic status and health: Current findings and future concerns. In G. J. Yoo, M. Le, \& A. Oda (Eds.), Handbook of Asian American health (pp. 173-183). New York, NY: Springer.

Zhang, J., \& Norvilitis, J. M. (2002). Measuring Chinese psychological well-being with Western developed instruments. Journal of Personality Assessment, 79(3), 492-511. doi:10.1207/S153277 52IPA7903_06. 